\title{
Descemet membrane endothelial keratoplasty with stromal rim (DMEK-S) in complicated patients
}

\author{
Pavel Studeny, Miroslav Veith, Deli Krizova
}

\begin{abstract}
Purpose. To evaluate the outcomes of the hybrid technique of posterior lamellar keratoplasty (Descemets' membrane endothelial keratoplasty with stromal rim, DMEK-S) in eyes with multiple ocular pathologies.

Methods. A retrospective case control study of the consecutive group of all eyes with combined ocular pathologies which underwent DMEK-S keratoplasty. We evaluated the number of pre- and post-operative complications; uncorrected distance visual acuity (UDVA); corrected distance visual acuity (CDVA) and endothelial cell density (ECD).

The first group (Group 1 - 100 eyes) was compared to a control group of eyes (Group 2 - 145 eyes) without combined ocular pathologies, which were operated throughout the same time period. Comparisons were made in the $12^{\text {th }}$ month of the follow-up.

Results. Both the UDVA and the CDVA statistical values 12 months after the surgery were significantly better in Group 2. ECD was comparable in both groups. The number of complications during surgery was considerably higher in Group 1 , where a certain type of complication occurred in 15 eyes (15\%), while in Group 2 this complication occurred only in 9 eyes (6.2\%). In Group 1 a slightly higher number of rebubbling cases (52\% vs. $48.3 \%$ ) occurred, as well as more than twice as many cases of primary graft failure (34\% vs. 15.7\%) compared to Group 2.

Conclusion. DMEK-S - hybrid endothelial transplantation in complicated eyes in the evaluated group of patients led to a noticeable improvement of visual acuity. When compared to surgeries in uncomplicated eyes, DMEK-S results in a higher rate of postoperative complications, especially graft detachment and primary failure.
\end{abstract}

Key words: DMEK-S, lamellar keratoplasty, endothelial dystrophy, endothelial transplantation

Received: April 10, 2018; Revised: July 7, 2018; Accepted: September 13, 2018; Available online: September 20, 2018 https://doi.org/10.5507/bp.2018.056

(c) 2019 The Authors. This is an open access article licensed under the Creative Commons Attribution License (https://creativecommons.org/licenses/by/4.0/).

Department of Ophthalmology, Kralovske Vinohrady Teaching Hospital, Srobarova 50, 10034, Prague, Czech Republic Corresponding author: Pavel Studeny, e-mail: studenypavel@seznam.cz

\section{INTRODUCTION}

There are currently several types of lamellar endothelial transplantation available for patients with endothelial dysfunction, such as bullous keratophaty, Fuchs endothelial dystrophy, posterior polymorphous endothelial dystrophy, iridocorneal endothelial syndrome, and corneal endothelial failure after perforating keratoplasty (PK) or lamellar keratoplasty.

The two most frequently used techniques of lamellar endothelial transplantation are DSAEK (Descemet stripping automated endothelial keratoplasty) where the lamella consists of deep stromal layers, Descemet's membrane (DM), and endothelium ${ }^{1}$; and DMEK (Descemet's membrane endothelial keratoplasty) where the lamella consists only of DM and endothelium ${ }^{2,3}$.

Since 2007 the most commonly used method within our department has been the DMEK-S method, where a hybrid lamella is transplanted. Hybrid lamella consists of DM and endothelium in its central part, without the presence of stroma, while in peripheral areas there is a rim of deep stromal layers. The big-bubble technique was used for the DM separation ${ }^{4}$. The hybrid lamella theoretically combines the advantages of both previously mentioned techniques, i.e. a relatively easy manipulation (DSAEK) and an outstanding postoperative visual acuity after successfully performed surgeries (DMEK) (ref. ${ }^{5-8}$ ).

A lot of studies have evalutated the results of these techniques, however the authors usually compare the data of uncomplicated patients, such as eyes with no other ocular pathology than endothelial dysfunction. Though many of these patients indicated for the endothelial transplantation stated, that they had previously suffered from other eye diseases or underwent some other eye surgeries.

Performing a surgery on eyes affected in this manner is often more demanding than the standard techniques and therefore may bring multiple complications, both during surgery and in the postoperative period.

The aim of this study is to compare the results of DMEK-S (visual acuity, frequency of complications during and after surgery) in complicated and uncomplicated patients.

\section{MATERIALS AND METHODS}

This study is a retrospective evaluation of a consecutive group of all patients with combined ocular pathologies who underwent DMEK-S keratoplasty at the Ophthalmology Department of Kralovske Vinohrady 
Teaching Hospital and the 3rd Medical Faculty of Charles University, Prague, from January 2007 to March 2015.

The first group of eyes (Group 1) was compared to the second group (Group 2), which was operated throughout the same time period, but without combined ocular pathologies influencing the surgery, the postoperative process of healing, and the postoperative visual acuity.

In the Group 1 we also individually evaluated results in subgroups of eyes ( $\mathrm{n} \geq 10$ eyes) with different eye conditions - after pars plana vitrectomy (PPV), with anterior chamber intraocular lens (AC IOL), eyes after PK, eyes after glaucoma surgery and eyes with pseudoexfolative syndrome (PEX).

We evaluated the number of intra- and post-operative complications; uncorrected distance visual acuity (UDVA) and corrected distance visual acuity (CDVA) in decimal values; endothelial cell density (ECD) in $\mathrm{cc} / \mathrm{mm}^{2}$.

Differences between the two groups, as well as differences between individual sub-groups and Group 1, were evaluated using Student's unpaired t-test. Differences with $P \leq 0.05$ were evaluated as statistically significant.

Table 1. Overview of types and rates of ocular pathologies in Group 1.

\begin{tabular}{lc}
\hline Eye after pars plana vitrectomy & 30 \\
Anterior chamber intraocular lens & 21 \\
Pseudoexfoliation syndrome & 15 \\
Eye after glaucoma surgery & 12 \\
Eye after perforating keratoplasty & 11 \\
Aphakia & 7 \\
Subluxation or luxation of intraocular lens & 6 \\
Diabetic retinopathy & 5 \\
Corneal leucoma & 5 \\
Myopia gravis & 4 \\
Microphthalmus & 1 \\
Megalocornea & 1 \\
Vitreous fibres in anterior chamber & 1 \\
\hline
\end{tabular}

Postoperative results are summarised in Table 2. Twelve months after surgery the UDVA was $0.25 \pm 0.21$, the CDVA was $0.45 \pm 0.29$, and the ECD was $1483 \pm 675 \mathrm{cc} / \mathrm{mm}^{2}$, which represents a decrease of $49 \pm 23 \%$.
Evaluations were always made in the $12^{\text {th }}$ month of follow-up because surgery results were relatively stable by that time and the groups were sufficiently representative.

\section{RESULTS}

Group 1 examined 100 eyes of 66 patients with the mean age of $69 \pm 14$ years (minimum age was 22 years; maximum age was 93 years). Types of ocular pathologies recorded in Group 1 are described in detail in Table 1. There were more than one ocular pathologies in some eyes, which were included in several sub-groups evaluating the individual risk factors.

Different types of complications occurred in 15 eyes during the surgery: graft turning over, bleeding, loosening of lens ligament, flat anterior chamber, herniation of vitreous body, presence of silicon oil in anterior chamber, lamella graft in vitreous cavity, miosis.

From various postoperative complications in Group 1 graft detachment occurred in 52 eyes (52\%). In these 52 eyes the rebubbling was performed once in 29 eyes, twice in 15 eyes, three times in 5 eyes, and four times in 3 eyes. In $34 \%$ of the cases a primary graft failure occurred, resulting in the absence of postoperative corneal clearance.

In 4 cases a repeated endothelial dysfunction and corneal oedema occurred after a period of successful corneal clearance (secondary graft failure).

Postoperative increase of intraocular pressure was recorded in 11 eyes, exceeding the value of $24 \mathrm{mmHg}$. In 7 out of these 11 eyes the intraocular pressure continued to increase even after completing the steroid therapy. Therefore, this condition was identified as a secondary glaucoma developed de novo (2 eyes) or as a secondary decompensated glaucoma of a different type, already present in the preoperative phase: juvenile glaucoma (1 eye), pseudoexfoliative glaucoma ( 1 eye), primary open-angle glaucoma ( 1 eye), and secondary glaucoma after different eye surgery ( 2 eyes).

In the treatment of this glaucoma a change in conservative therapy was sufficiently effective in 5 patients, while in 2 patients the glaucoma surgery using Ex-Press

Table 2. Comparison of results preoperatively and 12 months after surgery.

\begin{tabular}{lccccccc}
\hline & Group 1 & PPV & AC IOL & PEX & GS & PK & Group 2 \\
\hline $\mathrm{n}$ & 100 & 30 & 21 & 15 & 12 & 11 & 145 \\
UDVA preop. & $0.07 \pm 0.11$ & $0.03 \pm 0.04$ & $0.08 \pm 0.12$ & $0.09 \pm 0.13$ & $0.06 \pm 0.09$ & $0.05 \pm 0.05$ & $0.16 \pm 0.17$ \\
UDVA postop. & $0.25 \pm 0.21$ & $0.20 \pm 0.13$ & $0.20 \pm 0.20$ & $0.41 \pm 0.24$ & $0.16 \pm 0.21$ & $0.13 \pm 0.09$ & $0.58 \pm 0.26$ \\
CDVA preop. & $0.11 \pm 0.16$ & $0.07 \pm 0.15$ & $0.12 \pm 0.16$ & $0.14 \pm 0.20$ & $0.09 \pm 0.11$ & $0.12 \pm 0.16$ & $0.20 \pm 0.20$ \\
CDVA postop. & $0.45 \pm 0.29$ & $0.40 \pm 0.34$ & $0.41 \pm 0.24$ & $0.55 \pm 0.26$ & $0.29 \pm 0.30$ & $0.35 \pm 0.23$ & $0.81 \pm 0.22$ \\
ECD preop. & $2874 \pm 296$ & $2862 \pm 257$ & $2862 \pm 263$ & $2830 \pm 379$ & $2921 \pm 304$ & $3024 \pm 302$ & $2859 \pm 280$ \\
ECD postop. & $1483 \pm 675$ & $1674 \pm 570$ & $1638 \pm 662$ & $1889 \pm 603$ & $1595 \pm 849$ & $818 \pm 96$ & $1187 \pm 508$ \\
Loss (\%) & $49 \pm 23$ & $39 \pm 21$ & $39 \pm 24$ & $40 \pm 14$ & $47 \pm 27$ & $73 \pm 3$ & $60 \pm 17$ \\
Rebubbling (\%) & 52 & 53.3 & 42.8 & 46.7 & 58.3 & 60 & 48.3 \\
PF (\%) & 34 & 46.7 & 42.8 & 40.0 & 16.7 & 30 & 22 \\
\hline
\end{tabular}

$\mathrm{UDVA}=$ uncorrected distance visual acuity; $\mathrm{CDVA}=$ corrected distance visual acuity; $\mathrm{ECD}=$ endothelial cell density $\left(\mathrm{cc} / \mathrm{mm}{ }^{2}\right), \mathrm{PF}=$ primary failure PPV = pars plana vitrectomy, AC IOL = anterior chamber intraocular lens, $\mathrm{PEX}=$ pseudoexfoliative syndrome, GS = glaucoma surgery, $\mathrm{PK}=$ perforating keratoplasty 
mini glaucoma shunt was performed (Alcon Laboratories, Fort Worth, TX, USA).

Transplant rejection occurred in 1 patient, 18 months after surgery.

A number of other complications occurred in Group 1, always in 1 patient. These were: mild keratitis, epiretinal membrane, retrocorneal membrane, pupiloplegia, cystoid macular edema, subluxation of intraocular lens (IOL), newly developed wet form of age-related macular degeneration, cataract, branch retinal vein occlusion, luxation of IOL into vitreous cavity, and candida corneal ulcer (due to overall immune deficiency). The frequencies of postoperative complications in Group 1 and 2 are summarised in Table 3 .

In the comparative Group 2 were 145 eyes with single ocular pathology of 102 patients with the mean age of 72 \pm 10 years (minimum age was 42 years; maximum age was 91 years).

A certain type of complication occurred in 9 eyes during surgery: graft turning over in 6 eyes, pronounced intraoperative floppy iris syndrome (IFIS) in 1 eye, shallow anterior chamber in 1 eye, and in 1 eye a reimplantation of the lamellae due to the graft being pushed out of the anterior chamber by irrigation flow.

Postoperative results are summed up in Table 2. Twelve months after surgery UDVA was $0.58 \pm 0.26$, CDVA was $0.81 \pm 0.22$, ECD was $1187 \pm 508 \mathrm{bb} / \mathrm{mm}^{2}$ which represents a decrease of $60 \pm 17 \%$.

From various postoperative complications in Group 2 a graft detachment occurred in 70 eyes (48.3\%). Out of these 70 eyes the rebubbling was performed once in 51 eyes, twice in 11 eyes, three times in 6 eyes, and four times in 2 eyes. In 22 cases ( $15.7 \%$ ) a primary graft failure occurred, while secondary graft failure occurred in 5 cases: in one case after 3 months, in two cases after 6 months, in two cases after 12 months.

In 12 eyes with normal preoperative values of intraocular pressure, a postoperative increase in the intraocular pressure was recorded. In 10 eyes intraocular pressure increase continued, even after completing the steroid therapy. Therefore, this condition was identified as a secondary glaucoma. In 2 cases the glaucoma surgery was performed- deep sclerectomy with T-Flux implant (Carl Zeiss Meditec, Germany).

Other postoperative complications included: transplant rejection in 2 eyes, one 3 months, and one 12 months after surgery.
Nd: YAG laser capsulotomy was necessary in 4 eyes due to posterior capsular opacification, and a cataract developed in 1 phakic eye, recommended for surgery.

When comparing Group 1 (complicated patients) with Group 2 (uncomplicated patients), both UDVA and CDVA values 12 months after surgery were statistically significantly better in Group 2. ECD was comparable in both groups.

As expected, the number of perioperative complications were considerably higher in Group 1 where a certain type of complication occurred in 15 eyes (15\%), while in Group 2 only in 9 eyes (6.2\%).

In Group 1 a slightly higher (statistically insignificant) number of rebubbling cases (52\% vs. $48.3 \%$ ) occurred, as well as more than twice as many cases of primary graft failure (34\% vs. $15.7 \%$, statistically significant) compared to Group 2. The numbers of the remaining types of complications were roughly the same in both Groups.

Visual acuity and endothelial loss 12 months after surgery in individual sub-groups of Group 1 were similar and comparable with the results for Group 1 as a whole. The only statistically significant difference was a higher endothelial loss in the sub-group of patients after PK $(P=0.0075)$. The rest of the differences were not statistically significant.

Among the postoperative complications a graft detachment with the necessity of rebubbling occurred most commonly in the sub-group of patients after PK (60\%) and after glaucoma surgery (58.3\%). Primary failure ocurred noticeably more often in the sub-group of patients with aphakia (71.4\%), while in the sub-group of patients after glaucoma surgery its percentage was the lowest (16.7\%), coming close to the results of the Group 2 with uncomplicated eyes $(15.7 \%)$. The frequencies of graft detachment and primary failure were similar in the rest of the sub-groups.

\section{DISCUSSION}

Results of endothelial transplantation in complicated patients are only rarely published and the numbers of patients involved are relatively limited. Most of the studies available are focused on DSAEK type of surgery and each study usually looks at a specific comorbidity.

Most of the published studies describe results and frequencies of complications in patients with AC IOL

Table 3. Comparing the numbers of individual postoperative complications in Groups 1 and 2.

\begin{tabular}{lcrcc}
\hline & \multicolumn{2}{c}{ Complicated eyes, $\mathrm{n}=100$} & \multicolumn{2}{c}{ Uncomplicated eyes, $\mathrm{n}=145$} \\
& Number & $\%$ & Number & \multicolumn{1}{c}{ \% } \\
\hline Rebubbling & 52 & 52 & 70 & 48.3 \\
Primary failure & 34 & 34 & 22 & 15.7 \\
Secondary failure & 4 & 4 & 12 & 3.4 \\
Increased IOP & 11 & 11 & 10 & 8.3 \\
Secondary glaucoma & 7 & 7 & 3 & 6.9 \\
Rejection & 1 & 1 & & 2.1 \\
\hline
\end{tabular}

$\mathrm{IOP}=$ Intraocular pressure 
or with aphakia. Some studies also include an exchange of the AC IOL for a posterior chamber intraocular lens (PC IOL).

Shah described results at six months after DSAEK with IOL exchange combined surgery in the group of 19 eyes. No dislocations or primary graft failures occurred; the mean CDVA value was 0.42 and mean donor cell loss at 6 months was $33 \%$ (ref. $^{9}$ ).

Similar group of patients with DSEK (Descemet stripping endothelial keratoplasty) combined with AC IOL removal and scleral fixation of a PC IOL was described by Wylegała. The study group consisted of 11 eyes and the follow-up was 19.3 months. Lamella detachment occurred in 3 eyes, corneal rejection was observed in 1 eye. The mean CDVA value was 0.36; mean ECD value was 2048 $\mathrm{bb} / \mathrm{mm}^{2}$. Endothelial cell loss was $36 \%$ (ref. $^{10}$ ).

Vélez, in a total of 9 aphakic eyes with simultaneous DSEK and retropupillary fixated iris claw lens (Artisan), states the minimum visual acuity of 0.25 at six months after the surgery ${ }^{11}$.

Hsu compared outcomes and difficulties of DSAEK in complicated cases with IOL exchange, aphakia, or AC IOL implants (30 eyes) with a group of uncomplicated DSAEK cases (109 eyes). Of the 30 complicated DSAEK cases, 14 eyes underwent IOL exchange, 5 AC IOLs were not removed, 5 eyes remained aphakic, and 5 eyes had IOL exchange done before or after DSAEK. One eye had an iris-supported phakic IOL removed, followed by cataract extraction with IOL implantation at the time of DSAEK. In group of complicated cases only $27.6 \%$ of patients achieved a CDVA value of 0.5 and better, compared to $94.4 \%$ in the other group of patients without visually significant comorbidities. $60 \%$ of the complicated eyes achieved a CDVA value of 0.29 and better. Of the 30 complicated eyes, 5 (16.7\%) had graft detachments and 5 (16.7\%) developed IOL dislocations. Primary graft failure occurred in 3 cases (10\%) $\left(\right.$ ref. $\left.^{12}\right)$.

Ang evaluated an endothelial cell loss in 18 eyes, which underwent DSAEK while retaining AC IOL, and compared it to the results of a control group with PC IOL left in place. The endothelial cell loss at 1 year was $31.9 \pm 21.3 \%$ in the DSAEK with AC IOL group compared to $24.5 \pm 21.2 \%$ in the DSAEK group; however, this figure was significantly greater in the DSAEK with AC IOL group at 3 years compared to the DSAEK group $(55.3 \pm 29.2 \%$ vs $33.3 \pm 20.8 \%)$ (ref. ${ }^{13}$ ).

Elderkin evaluated the frequency of complications in a group of 11 patients with corneal decompensation and AC IOL who underwent DSAEK. Six patients had a temporary suture to secure their graft. There was 1 graft dislocation postoperatively and 1 primary graft failure among those 11 patients ${ }^{14}$.

Most authors agree that the results in complicated patients are slightly worse and the frequency of complications is higher, when compared to the results in eyes without other ocular copathology.

Therefore, it is rather rare to see results such as those published by Shpitzer, who retrospectively compared patients who underwent DSAEK and had a retained AC IOL, with patients who underwent DSAEK and had a PC
IOL. There were 11 eyes with an AC IOL and the authors found no significant differences between the groups in terms of perioperative complications, change in visual acuity, graft survival, and endothelial cell loss ${ }^{15}$.

In our sub-group of patients with $\mathrm{AC}$ IOL the mean visual acuity at 12 months was $0.41 \pm 0.24$, thus comparable or even better than in published studies. This difference can be explained by generally better results for visual acuity after DMEK type of surgery compared to DSAEK and DSEK.

On the other hand, a significantly higher frequency of postoperative complications was recorded, especially of graft detachment (42.8\% in eyes with AC IOL and 57.1\% in aphakic eyes) and primary graft failure $(42.8 \%$ and $71.4 \%$, respectively). Furthermore, endothelial cell loss was slightly higher in our group compared to published results $(39 \pm 24 \%)$.

In the case of aphakic eyes our method, i.e. DMEK-S combined with IOL implantation, turned out to be quite risky. It would probably be more appropriate to separate the two surgeries: perform IOL implantation first and the endothelial transplantation after that as a separate surgery, because of the lower frequency of complications in the sub-group of patients with AC IOL.

A group of 20 vitrectomized eyes selected for DMEK was described by Yoeruk. Seven eyes had a history of anterior vitrectomy and 14 eyes of PPV. Twelve months after surgery the CDVA was 0.25 and ECD was $1241 \pm$ $155 \mathrm{bb} / \mathrm{mm}^{2}$ on average ${ }^{16}$.

In our sub-group of patients after PPV mean CDVA after a 12-month follow-up was significantly better $(0.40 \pm 0.34)$, while ECD was lower $\left(1097 \pm 631 \mathrm{bb} / \mathrm{mm}^{2}\right)$. A specific type of complication in eyes after PPV, which was also once recorded in our sub-group, would be the unwanted dislocation of the donor graft into the posterior segment.

A series of this complication associated with DSAEK was described by Afshari et al. In 6 of 8 eyes PPV was used to remove the dislocated graft. Their study concluded that better postoperative results seem to be associated with the prompt removal of the posteriorly dislocated graft $^{17}$.

Mitry et al. evaluated results of patients who underwent DSAEK after failed PK. It was a multicentre study that included 246 eyes with the median follow-up of 17 months. In total, 19.1\% (47 of 246) of DSAEK grafts failed. Authors identified the following significant preoperative risk factors for failure: young recipient age, previous filtration surgery, and rejection episodes before PK failure. Authors came to conclusion that DSAEK after failed PK combines greater wound stability and reduces suture-related complications, with visual outcomes and graft survival rates comparable to those of a second PK (ref. ${ }^{18}$ ).

Chaurasia notes primary graft failure in $22.2 \%$ and visual acuity of 0.33 and better in $63 \%$ of eyes in a group of 27 eyes with DSEK for failed penetrating keratoplasty (PK) $\left(\right.$ ref. $\left.^{19}\right)$.

Lee described results of a group of 8 eyes with DSAEK after PK. Seven of the patients showed an improvement in CDVA, while the average CDVA acuity 6 months post- 
operatively was 0.1 . Graft detachment occurred in 4 eyes $(50 \%)$ and 1 eye experienced graft failure ${ }^{20}$.

Covert published similar results in 7 patients: average visual acuity was 0.31 ; graft detachment occurred in 3 eyes and primary failure in 3 eyes $\left(42.9 \%\right.$ ) $\left(\right.$ ref. ${ }^{21}$ ).

In our sub-group of patients after PK a relatively good visual acuity was achieved $(0.35 \pm 0.23)$, however endothelial loss in this sub-group was by far the highest among all studied types of comorbidities $(2180 \pm 203)$. It was even statistically significantly higher than that for the whole of Group 1. This is probably also related to a higher rate of postoperative complications in our sub-group: $60 \%$ of graft detachments and $30 \%$ of primary failures.

\section{CONCLUSION}

DMEK-S - hybrid endothelial transplantation in complicated eyes led in our group of patients to a marked improvement of visual acuity. After successful surgeries the resulting visual acuity is higher than that in published data for comparable patients after DSAEK.

When compared with surgeries in uncomplicated eyes and in complicated eyes after DSAEK, DMEK-S results in a high rate of postoperative complications, especially graft detachment and primary failure.

In aphakic eyes we consider DMEK-S combined with AC IOL implantation as unsuitable.

When choosing the optimal surgery technique, the surgeon must always individually evaluate possible pros and cons of a given type of surgery.

\section{ABBREVIATIONS}

AC IOL, anterior chamber intraocular lens; CDVA, corrected distance visual acuity; DM, Descemet's membrane; DMEK, Descemet's membrane endothelial keratoplasty; DMEK-S, Descemet 's membrane endothelial keratoplasty with stromal rim; DSAEK, Descemet stripping automated endothelial keratoplasty; DSEK, Descemet stripping endothelial keratoplasty; ECD, endothelial cell density; IOL, intraocular lens; PC IOL, posterior chamber intraocular lens; PK, perforating keratoplasty; PPV, pars plana vitrectomy; UDVA, uncorrected distance visual acuity.

Author contributions: PS: literature search; PS, MV: manuscript writing; DK: data collection; PS, MV, DK: data analysis, final approval

Conflict of interest statement: The authors state that there are no conflicts of interest regarding the publication of this article.

\section{REFERENCES}

1. Gorovoy MS. Descemet-stripping automated endothelial keratoplasty. Cornea 2006;25:886-9.
2. Melles GR, Ong TS, Ververs B, van der Wees J. Descemet membrane endothelial keratoplasty (DMEK). Cornea 2006;25:987-90.

3. Ham L, Dapena I, van Luijk C, van der Wees J, Melles GR. Descemet membrane endothelial keratoplasty (DMEK) for Fuchs endothelial dystrophy: review of the first 50 consecutive cases. Eye (Lond) 2009;23:1990-8.

4. Anwar M, Teichmann KD. Big-bubble technique to bare Descemet's membrane in anterior lamellar keratoplasty J Cataract Refract Surg 2002;28:398-403.

5. McCauley MB, Price FW Jr, Price MO. Descemet membrane automated endothelial keratoplasty: hybrid technique combining DSAEK stability with DMEK visual results. J Cataract Refract Surg 2009;35:1659-64.

6. Pereira Cda R, Guerra FP, Price FW Jr., Price MO. Descemet's membrane automated endothelial keratoplasty (DMAEK): visual outcomes and visual quality. J Ophthalmol 2011;95:951-4.

7. Studeny P, Farkas A, Vokrojova M, Liskova P, Jirsova K. Descemet membrane endothelial keratoplasty with a stromal rim (DMEK-S). Br J Ophthalmol 2010;94:909-14.

8. Studeny P, Sivekova D, Liehneova K, Vokrojova M., Kuchynka P. Hybrid Technique of Lamellar Keratoplasty (DMEK-S). J Ophthalmol 2013;2013:254383.

9. Shah AK, Terry MA, Shamie N, Chen ES, Phillips PM, Hoar KL, Friend DJ, Davis-Boozer D. Complications and clinical outcomes of descemet stripping automated endothelial keratoplasty with intraocular lens exchange. Am J Ophthalmol 2010;149:390-7.

10. Wylegała E, Tarnawska D. Management of pseudophakic bullous keratopathy by combined Descemet-stripping endothelial keratoplasty and intraocular lens exchange. J Cataract Refract Surg 2008;34:1708-14.

11. Vélez FM, Mannis MJ, Izquierdo L Jr., Sánchez JG, Velásquez LF, Rojas S. Simultaneous surgery for corneal edema and aphakia: DSEK and placement of a retropupillary iris claw lens. Cornea 2014;33:197-200.

12. Hsu M, Jorgensen AJ, Moshirfar M, Mifflin MD. Management and outcomes of descemet stripping automated endothelial keratoplasty with intraocular lens exchange, aphakia, and anterior chamber intraocular lens. Cornea 2013;32:64-8.

13. Ang M, Li L, Chua D, Wong C, Hton HM, Mehta JS, Tan D. Descemet's stripping automated endothelial keratoplasty with anterior chamber intraocular lenses: complications and 3-year outcomes. $\mathrm{Br} \mathrm{J}$ Ophthalmol 2014;98:1028-32.

14. Elderkin S, Tu E, Sugar J, Reddy S, Kadakia A, Ramaswamy R, Djalilian A. Outcome of descemet stripping automated endothelial keratoplasty in patients with an anterior chamber intraocular lens. Cornea 2010;29:1273-7.

15. Shpitzer SA, Rosenblatt A, Bahar I. Outcomes of descemet stripping automated endothelial keratoplasty in patients with an anterior chamber versus posterior chamber intraocular lens. Cornea 2014;33:686-90.

16. Yoeruek E, Rubino G, Bayyoud T, Bartz-Schmidt KU. Descemet membrane endothelial keratoplasty in vitrectomized eyes: clinical results. Cornea 2015;34:1-5.

17. Afshari NA, Gorovoy MS, Yoo SH, Kim T, Carlson AN, Rosenwasser GO, Griffin NB, McCuen BW 2nd, Toth CA, Price FW Jr, Price M, Fernandez MM. Dislocation of the donor graft to the posterior segment in descemet stripping automated endothelial keratoplasty. Am J Ophthalmol 2012;153:638-42.

18. Mitry D, Bhogal M, Patel AK, Lee BS, Chai SM, Price MO, Price FW Jr, Jun AS, Aldave AJ, Mehta JS, Busin M, Allan BD. Descemet stripping automated endothelial keratoplasty after failed penetrating keratoplasty: survival, rejection risk, and visual outcome. JAMA Ophthalmol 2014;132:742-9.

19. Chaurasia S, Murthy S, Ramappa M, Mohamed A, Garg P. Outcomes of Descemet's stripping endothelial keratoplasty in eyes with failed therapeutic penetrating keratoplasty. Acta Ophthalmol 2014;92:16770.

20. Lee BS, Stark WJ, Jun AS. Descemet-stripping automated endothelial keratoplasty: a successful alternative to repeat penetrating keratoplasty. Clin Experiment Ophthalmol 2011;39:195-200.

21. Covert DJ, Koenig SB. Descemet stripping and automated endothelial keratoplasty (DSAEK) in eyes with failed penetrating keratoplasty. Cornea 2007;26:692-6. 